\title{
Organic coprecipitates with calcite: NMR spectroscopic evidence
}

\author{
Brian L. Phillips*, Young J. Lee, Richard J. Reeder \\ Center for Environmental Molecular Science, Department of Geosciences, \\ State University of New York, Stony Brook, NY 11794-2100
}

\section{Supporting Information:}

6 Pages

3 Figures

*Corresponding author. Phone: (631)-632-6853. Fax: (631)-632-6840.

E-mail: brian.phillips@sunysb.edu 
NMR Spectroscopy. The solid-state ${ }^{13} \mathrm{C}$ cross-polarization (CP-MAS) NMR spectra were measured on a $400 \mathrm{MHz}$ Varian Inova spectrometer operating at $100.6 \mathrm{MHz}$, using a Varian/Chemagnetics "T3" probe assembly configured for $7.5 \mathrm{~mm}$ (outside diameter) rotors with a spinner housing constructed to yield a very low ${ }^{13} \mathrm{C}\left\{{ }^{1} \mathrm{H}\right\}$ CP-MAS background signal. The ${ }^{13} \mathrm{C}\left\{{ }^{1} \mathrm{H}\right\}$ CP-MAS spectra were obtained with standard pulse sequences at spinning rates of 3$5 \mathrm{kHz}$, with $8 \mu$ s excitation pulse $\left(90^{\circ}\right)$ and 0.5-2 ms contact time, during which the ${ }^{1} \mathrm{H} \mathrm{B}_{1}$ field was ramped approximately $\pm 10 \mathrm{kHz}$ about the optimum match condition. Observation of signal from the coprecipitated organic molecules (samples cc3101, cc387, cc4003) required 1-2 days of data collection at relaxation delays of $0.5-1.0 \mathrm{~s}$ (175,000 to 320,000 total acquisitions). Contact times of 1-2 ms yield maximum CP-MAS signal intensity for the organic ligands. Chemical shifts are reported relative to tetramethylsilane (TMS), but measured relative to a secondary sample of adamantane (38.6 ppm).

For all of our calcite samples, we measured a carbonate chemical shift $(168.7 \mathrm{ppm})$ that differs consistently by $+1.2 \mathrm{ppm}$ from those reported in a previous study (1). A similar difference was noted for a sample of aragonite: 171.2 ppm measured vs. 169.9 ppm from Ref. (1). For the 3:2 Ca(II)-citrate tetrahydrate (Aldrich) we found chemical shifts of 185.6, 189.7, $181.0,180.6$, and $179.7 \mathrm{ppm}$ for the carboxylate region, and 75.6 for the central C-OH.

The cross-polarization dynamics for the calcite carbonate resonance was measured for samples cc3107, cc3109, and cc3113, which were prepared from ${ }^{13} \mathrm{C}$-labeled sodium carbonate (Aldrich Isotec). The CP-MAS intensities were measured under identical experimental conditions at contact times from 0.2 to $16 \mathrm{~ms}$ and a constant spinning rate of $5.0 \mathrm{kHz}$. The intensity values were normalized by mass between samples by comparing intensities obtained for all three samples under identical conditions ( $4 \mathrm{~ms}$ contact time, $3 \mathrm{kHz}$ spinning rate, relaxation 
delay greater that $3 \cdot T_{1, \mathrm{H}}$ ), with the samples contained in the central one-third of the rotor volume to minimize variations in $\mathrm{B}_{1}$ homogeneity. We used ${ }^{13} \mathrm{C}$-detected inversion-recovery nullpoint method to estimate the $T_{1, \mathrm{H}}$ values for each sample, which gave $T_{1, \mathrm{H}}=0.9 \mathrm{~s}$ for $\operatorname{cc} 3107$ and cc3113, but $9.5 \mathrm{~s}$ for the sample prepared in $\mathrm{D}_{2} \mathrm{O}(\operatorname{cc} 3109)$.

${ }^{1} \mathrm{H}$ single-pulse (SP) MAS-NMR spectra were obtained on the same spectrometer system, using a Chemagnetics sample probe assembly configured for $4 \mathrm{~mm}$ rotors and modified to yield a low ${ }^{1} \mathrm{H}$ background signal. Samples were spun at $15.5 \mathrm{kHz}$, with the sample temperature maintained at $25^{\circ} \mathrm{C}$. Absolute ${ }^{1} \mathrm{H}-\mathrm{NMR}$ intensities were estimated by comparing the value of the first point of the time-domain data with that for a sample of analcime obtained under the same conditions. The time-domain data were adjusted by recalculating the first points and applying a phase correction to yield a pure absorption mode frequency-domain spectrum with a flat baseline. The time-domain origin was adjusted to require only a zero-order (frequency independent) phase correction. Chemical shifts are reported relative to TMS, via an external standard of hydroxylapatite for ${ }^{1} \mathrm{H}(\delta \equiv 0.2 \mathrm{ppm})$.

The ${ }^{1} \mathrm{H}$ SP spectra of the samples prepared in $\mathrm{H}_{2} \mathrm{O}$ contain a strong peak for water near $5.3 \mathrm{ppm}$ (Fig. SI-1), plus much smaller peaks near $+7.4,1.6,1.1$, and $0.2 \mathrm{ppm}$. The width of the peak at $5.3 \mathrm{ppm}$ suggests the presence of some restricted motion, but is too large to attribute to fluid inclusions. The peaks between 1.6 and $0.2 \mathrm{ppm}$ are present in all samples, including those prepared in $\mathrm{D}_{2} \mathrm{O}$, which suggests that they might be due primarily to the seed crystals. The peak at $7.4 \mathrm{ppm}$ could arise from bicarbonate protons and is exchangeable with the solvent water, as indicated by its absence in the spectrum of sample cc3109, which was synthesized in $\mathrm{D}_{2} \mathrm{O}$. Similar peaks were observed in a previous ${ }^{1} \mathrm{H}$ NMR study of phosphate sorption on calcite but could not be unambiguously assigned (2). All spectra also include a small, narrow peak for 
fluid-phase water at $4.8 \mathrm{ppm}$, accounting for less than $10 \%$ of the $\mathrm{H}$ in sample cc3113, which could arise from fluid inclusions and/or surface-adsorbed water; its presence in the spectrum of $\operatorname{cc} 3109\left(\mathrm{D}_{2} \mathrm{O}\right)$ suggests it arises primarily from fluid inclusions in the seed crystals. No rigorous attempt was made to remove surface adsorbed water, which we assume to be present at a similar level for all samples.

The 2-dimensional ${ }^{13} \mathrm{C}\left\{{ }^{1} \mathrm{H}\right\}$ heteronuclear correlation (HetCor) spectra were obtained at a spinning rate of $5.0 \mathrm{kHz}$. The pulse sequence used is a standard CP-MAS experiment with an incremented delay (T1) between the ${ }^{1} \mathrm{H}$ excitation and spin-locking pulse; no homonuclear ${ }^{1} \mathrm{H}$ decoupling was applied during T1. We collected 96-128 T1 slices, incremented to yield an $80 \mathrm{kHz}$ spectral window in $\mathrm{F} 1$, each corresponding to 320 acquisitions at relaxation delays of from 1 to $10 \mathrm{~s}$, depending on the sample. Contact times were those corresponding to maximum CP-MAS signal intensity, $2 \mathrm{~ms}$ for cc3107 and $10 \mathrm{~ms}$ for cc3109. However, we observed no significant change in the HETCOR spectrum of sample cc3107 upon changing the contact time to 0.7 or $10 \mathrm{~ms}$, except for a significantly poorer signal-to-noise ratio.

The 2-dimensional contour plots (Fig. SI-2) show intensity in F2 (the ${ }^{13} \mathrm{C}$-dimension) only at the chemical shift for carbonate $(+168.7 \mathrm{ppm})$ and its spinning sidebands. No resolution was observed across the ${ }^{13} \mathrm{C}$ resonance, so we could not determine from these data whether the different ${ }^{1} \mathrm{H}$ resonances correspond to hydrogen near distinct carbonate environments, or multiple $\mathrm{H}$ environments forming a single dipolar-coupled spin system. For this reason, the F1slices taken at the carbonate position in F2 (Fig. 4 in the text) contain all relevant information. These F1-slices correspond to the ${ }^{1} \mathrm{H}$ NMR spectra of the hydrogens from which magnetization is transferred to ${ }^{13} \mathrm{C}$ carbonate during cross-polarization. The indirectly detected ${ }^{1} \mathrm{H}$ signal corresponds to those protons coupled directly to the carbonate carbons plus those coupled via ${ }^{1} \mathrm{H}$ 
spin diffusion processes, because no homonuclear decoupling was applied to the ${ }^{1} \mathrm{H}$ spins during the $\mathrm{T} 1$ evolution period.

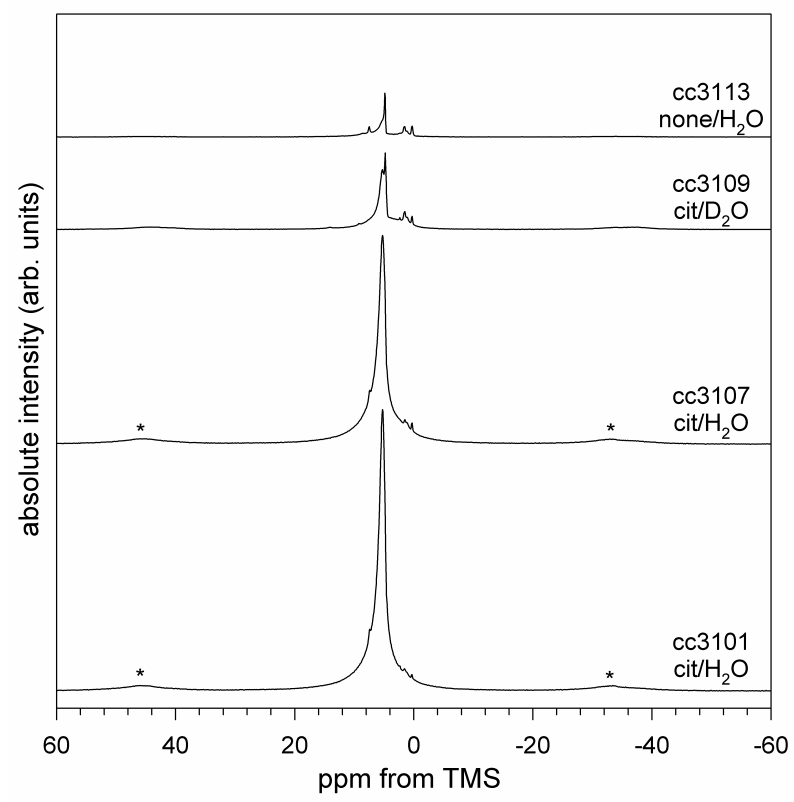

Figure SI-1. ${ }^{1}$ H MAS-NMR spectra for calcite samples of this study, after subtraction of the background signal measured for an empty rotor assembly. Spectral intensities are normalized to represent absolute integrated intensity per scan normalized by sample mass. Spectra acquired at $399.98 \mathrm{MHz}$ with $3 \mu$ s pulses $(\pi / 4)$, relaxation delays corresponding to $3 \mathrm{~T}_{1}$ 's (between 3 and $30 \mathrm{~s}$, depending on sample), for 200-800 acquisitions at a spinning rate of $15.5 \mathrm{kHz}$. Asterisks denote positions of the spinning sidebands.
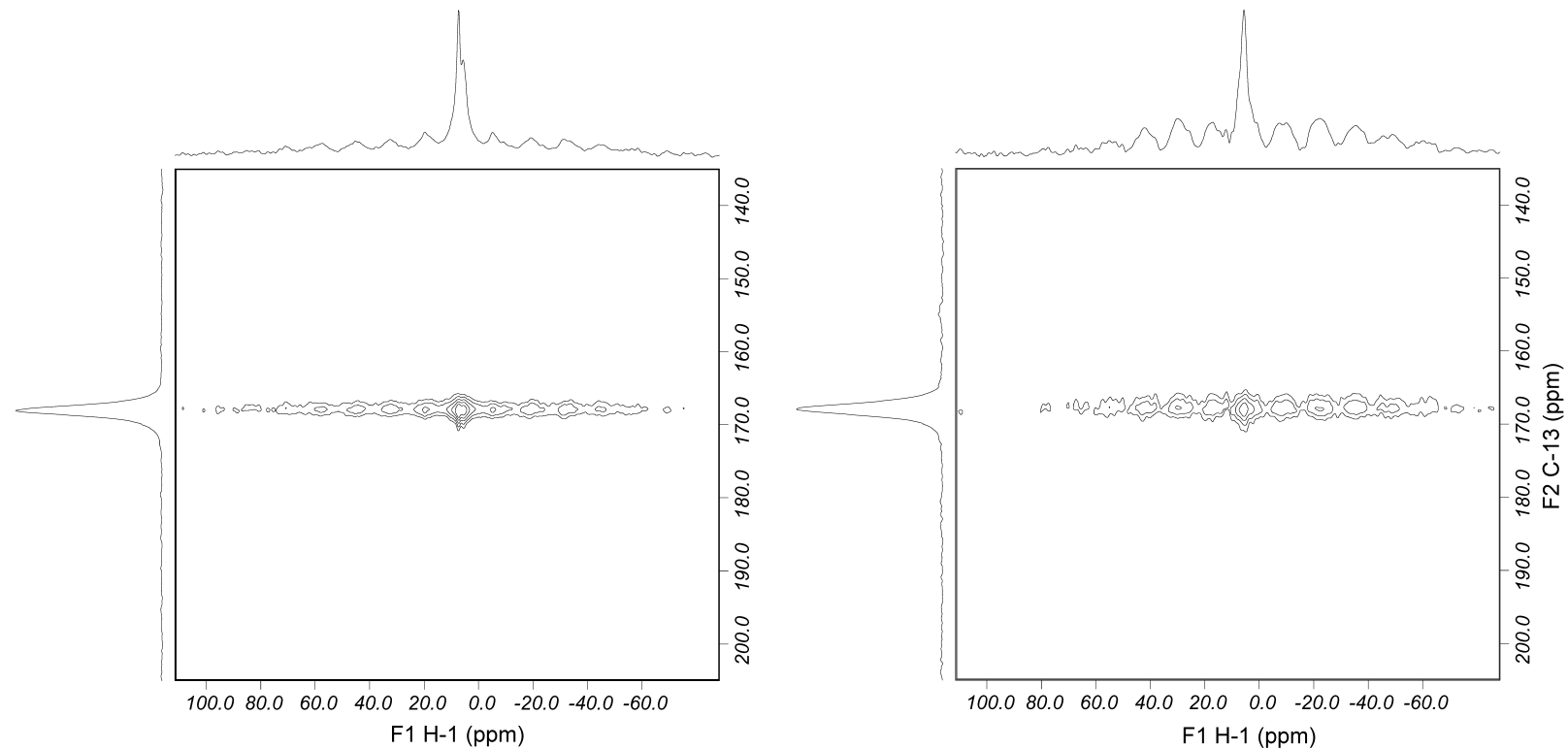

Figure SI-2. ${ }^{13} \mathrm{C}\left\{{ }^{1} \mathrm{H}\right\}$ HetCor spectra for samples cc3107 (left) and cc3109 (right). Contour levels are incremented geometrically starting at $7 \%$ to emphasize low-intensity peaks. Projections (top and left) are plotted in "skyline" mode. Spectra in Figure 4 (text) correspond to cross-sections taken at the carbonate centerband. 


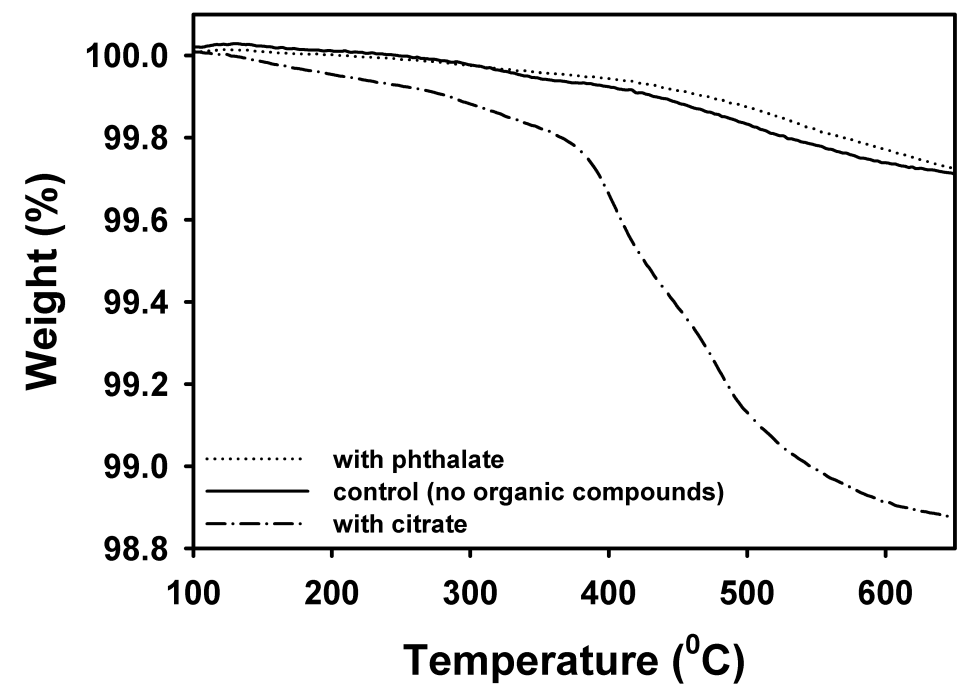

Figure SI-3. TGA weight-loss curves for calcite samples coprecipitated with phthalate (sample cc387) and citrate (cc3107), and for calcite precipitated under identical conditions but without added organic ligand. Heating rate was $5^{\circ} \mathrm{C} \mathrm{min}^{-1}$.

\section{Literature Cited}

(1) Papenguth, H. W.; Kirkpatrick, R. J.; Montez, B.; Sandberg, P. A., ${ }^{13}$ C MAS NMRspectroscopy of inorganic and biogenic carbonates. Am. Mineral. 1989, 74, 1152-1158.

(2) Hinedi, Z. R.; Goldberg, S.; Chang, A. C.; Yesinowski, J. P., A ${ }^{31}$ P and ${ }^{1}$ H MAS NMRstudy of phosphate sorption onto calcium-carbonate. J. Colloid Interf. Sci. 1992, 152, 141160. 